\title{
Dynamic Fuzzy Cognitive Models as a Means of Visual Analytics and Cognitive Graphics
}

\author{
Aleksei G. Massel \\ Melentiev Energy Systems Institute of Siberian Branch of the \\ Russian Academy of Sciences \\ Irkutsk, Russia \\ amassel@gmail.com
}

\begin{abstract}
The article discusses the concepts of visual analytics and cognitive graphics. The employment of cognitive models means and especially dynamic cognitive models is justified as a means of visualizing computational experiments in studies of the development directions of the energy sector from the standpoint of energy security problems. In addition to the above, dynamic cognitive maps are considered as an interaction interface with a model of the energy sector which will contribute to a significant acceleration of the computational experiment. The article provides an illustration example which displayed a relationship of dynamic cognitive maps and one of the energy sector mathematical models.
\end{abstract}

Keywords-semantic modeling methods, energy security, visual analytics, cognitive graphics, threat analysis.

\section{INTRODUCTION}

Nowadays more and more tools and research are aimed at presenting results using visualization. At the same time, attempts to use human cognitive psychology lead to a completely new qualitative assessment of information and data. Visual analytics is usually applied to the development of user interaction methods and interactive visual tools when working with large volumes of data, contributing to the rapid solution of some analytical problems. Analytical tasks are part of the fastest-growing of all areas of information technology application since the automatic analysis can solve some of these problems [1]. It is applicable in cases where the data is strictly formalized and criteria for their comparison are reasonable.

A completely different picture emerges in situations when we are dealing with the identification of implicit knowledge [2] in the absence of a large amount of data or current models. In the event that traditional methods of visual analytics based on the visualization of data and models are not suitable.

In the 1990s, Zenkin [3] proposed the cognitive graphics concept as a set of techniques and methods for figuratively representing problem conditions, which allows us to either immediately see a solution or get a hint for finding it. Professor D.A. Pospelov formulated three main tasks of cognitive computer graphics [4]:

\author{
Daria A. Gaskova \\ Melentiev Energy Systems Institute of Siberian Branch of the \\ Russian Academy of Sciences \\ Irkutsk, Russia \\ gaskovada@gmail.com
}

1. Creation of such models of knowledge representation, in which it would be possible to represent both objects characteristic of logical thinking and image-pictures with which figurative thinking operates with monotonous means.

2. Visualization of that human knowledge for which it is not yet possible to select textual descriptions.

3. The search for ways to move from observable image-patterns to the formulation of a hypothesis about those mechanisms and processes that are hidden behind the dynamics of the observed patterns.

In connection with these principles, the authors propose to consider semantic models as the development of cognitive graphics means and visual analytics.

\section{VISUAL ANALYTICS}

There are two similar terms that some experts currently consider synonyms. They are "Visual Data Mining" and "Visual Analytics".

The first of them appeared Visual Data Mining. About in 2000 in Europe Visual Data Mining began to call the integration of Data Mining methods with visualization technologies. One of the first scientific schools in this area took shape at Koblenz University (Germany). The definition of Visual Data Mining is given in [5].

Visual Analytics dates back to 2004. It was proposed by Jim Thomas, one of the authors of the book "Illuminating the Path: The Research and Development Agenda for Visual Analytics" [6], released as a policy paper by the Pacific Northwest National Laboratory, one of sixteen US Department of Energy laboratories. Visual Analytics is defined as the ability to think analytically, supported by a graphical interface in the book. The laboratory was reoriented to Visual Analytics by order of the US Department of Homeland Security to support the counter-terrorism threat program. After that it becomes the largest research center, almost completely specializing in various aspects of Visual Analytics, with branches in several countries. The laboratory of the Koblenz University became part of it later. 
Until now, the main means of Visual Analytics have been attributed, for the most part, to the processing and analysis of large amounts of data, which in our opinion is a rather narrow interpretation of this concept.

In Russia studies of cognitive graphics, the concept of cognitive graphics can be considered a synonym for Visual Analytics, with the only difference being that with the help of cognitive graphics we can display knowledge representation models. Keirn, Andrienko et al. in [7] described the development of Visual Analytics as integration of visual and automatic data analysis methods with database technology for scalable interactive decision support. At the same time, the key concept is "data" and the process of their analysis using visualization to obtain knowledge. This work presents the possibility of visual analytics development as a stage of visualization of knowledge representation models.

Scientific school, in which authors involved, actively use semantic models to formalize implicit knowledge [8]. Semantic models allow us to perform a qualitative analysis of the problem [9]. Moreover, the use of graphical representations of these models can be attributed specifically to the field of cognitive graphics. For instance, cognitive maps proved himself to be especially good in the process of energy experts working with them. They make it possible to display causal relationships between various objects and factors in certain situations.

The prerequisite for the cognitive approach development was the inapplicability of accurate models for the analysis and modeling of problem situations arising in the social and economic systems development. This is due to the need to take into account a large number of factors, many of which were difficult to formalize. Cognitive maps are graphs, and cognitive modeling is carried out by presenting the problematic situation of the system in the form of a visual figure [10].

As a result of these considerations, the authors propose further to move on to the stage of visual analytics based on the use of elements of cognitive graphics (Fig. 1).

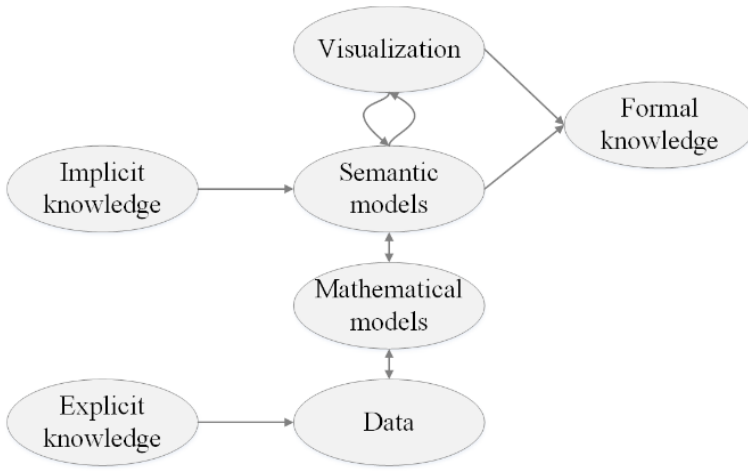

Fig. 1. Visual analytics of knowledge and data

It is proposed to visualize not only data but also to display individual elements of mathematical models that employ this data using semantic models. Example of this scheme performing is to use cognitive models. Notably, the use of traditional cognitive models is not a sufficient condition for displaying all aspects of the situation. In this case, we suggest connecting the means of dynamic cognitive models, which is described below.

\section{DYNAMIC COGNITIVE MAPS}

The cognitive map is a static essence and at one whack displays only the bond character of objects. However, modeling often requires a dynamic representation of the situation. In connection with this aspect, we proposed to consider dynamic cognitive maps.

A dynamic cognitive map differs from a regular cognitive map in that it is a bunch of cognitive maps that reflect the model at different points in time [11] (1):

$$
\{D\}=\left\{D_{t_{o}}, D_{t_{1}}, \ldots D_{t_{n}}\right\}
$$

where $\{D\}$ is a bunch of dynamic cognitive maps; $\left\{D_{t_{0}}\right\},\left\{D_{t_{1}}\right\},\left\{D_{t_{n}}\right\}$ are bunches of cognitive maps at points in time $\mathrm{t}_{0}, \mathrm{t}_{1}, \mathrm{t}_{\mathrm{n}}$.

The influence of objects on each other, depending on time, is described by a certain function called the weight of the connection $\mathrm{W}(\mathrm{t})$. In most cases, the weight of a connection in dynamic cognitive maps can be represented by simple functions. To present the mutual influence of various relationships on the same object, a bunch of parameters is introduced. Such parameters reflect the object's characteristics that are affected by incoming connections (2):

$$
\{P\}=\left\{P_{1}, P_{2}, \ldots P_{m}\right\}
$$

where $\{P\}$ is a bunch of parameters reflect the object's characteristics, $\left\{P_{1}, P_{2}, . . P_{m}\right\}$ are parameters, that are affected by incoming connections.

The object behavior itself or the occurrence of an event can be represented as a single impulse. This relationship reflects the period of the event occurrence equal to or less than the selected time step. Since the applied modeling domain is usually characterized by the complexity of the objects interdependencies and a large number of characteristics describing them, the behavior of an object can be described by a function and reflect the impact of objects over several time steps. The need to represent implicit relationships between concepts arises in case of changes in the parameters of the concepts in different time periods.

The advantages of dynamic cognitive maps in comparison with event modeling and cognitive maps are the ability to identify cause-effect relationships of concepts, and not only to analyse the development and consequences of the situation, modeling the relationships of concepts with respect to the timeline, as well as the ability to consider a separate part of the model i.e. an expert may limit modeling to 
consideration of individual concepts and their impact to each other [12].

\section{A. Dynamic Fuzzy Cognitive Maps}

At the current stage of the study, it is proposed to use cognitive maps for a qualitative analysis of energy security problems. The complexity of using dynamic cognitive maps in such a subject area is associated with the need for an expert to determine the connection weights and the speed of their influence on the model objects. In this connection, to simplify the expert's work with the model, it is proposed to introduce a linguistic scale that reflects the rate of change of weight and its effect on selected concepts.

\section{THE APPLICATION OF DYNAMIC FUZZY COGNITIVE MAPS IN ENERGY RESEARCH}

Currently, the methods for constructing the Dynamic Fuzzy Cognitive Maps are aimed at their integration with various mathematical models. In particular, the ESI SB RAS has developed a TRV simulation model, which is described in more detail in
[12], and the IT complex "INTEC-M" [13], which provides a computational experiment, to study the directions of energy development taking into account energy security aspects.

The following is a small example of the integration of the Dynamic Fuzzy Cognitive Maps and part of the TRV simulation model. The example includes part of the production structure of the electric power block of this model. The model includes the main types of power plants, types of fuel, the fuel consumption of each power plant, types of power plants, transport between regions and electricity consumption by consumers are indicated (Fig. 2).

\section{A. Modeling of Energy Security Threat "Lack of Investment"}

The "Lack of investment" threat and the impact of this threat on the "Aging of electricity-generating equipment" were selected as one of the illustrative examples. A similar relationship is generally characteristic of all energy systems. Figure 3 shows a fragment of the cognitive map "Lack of investment".

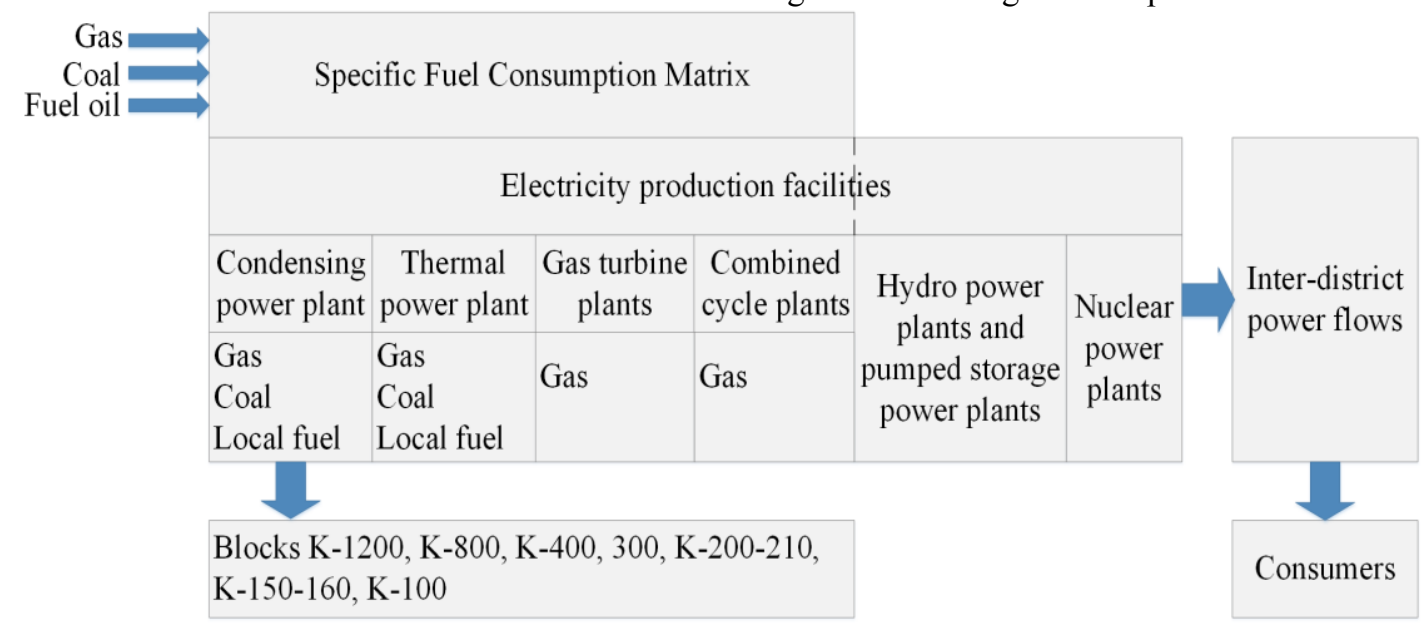

Fig. 2. Production structure of the TRV model electric power block

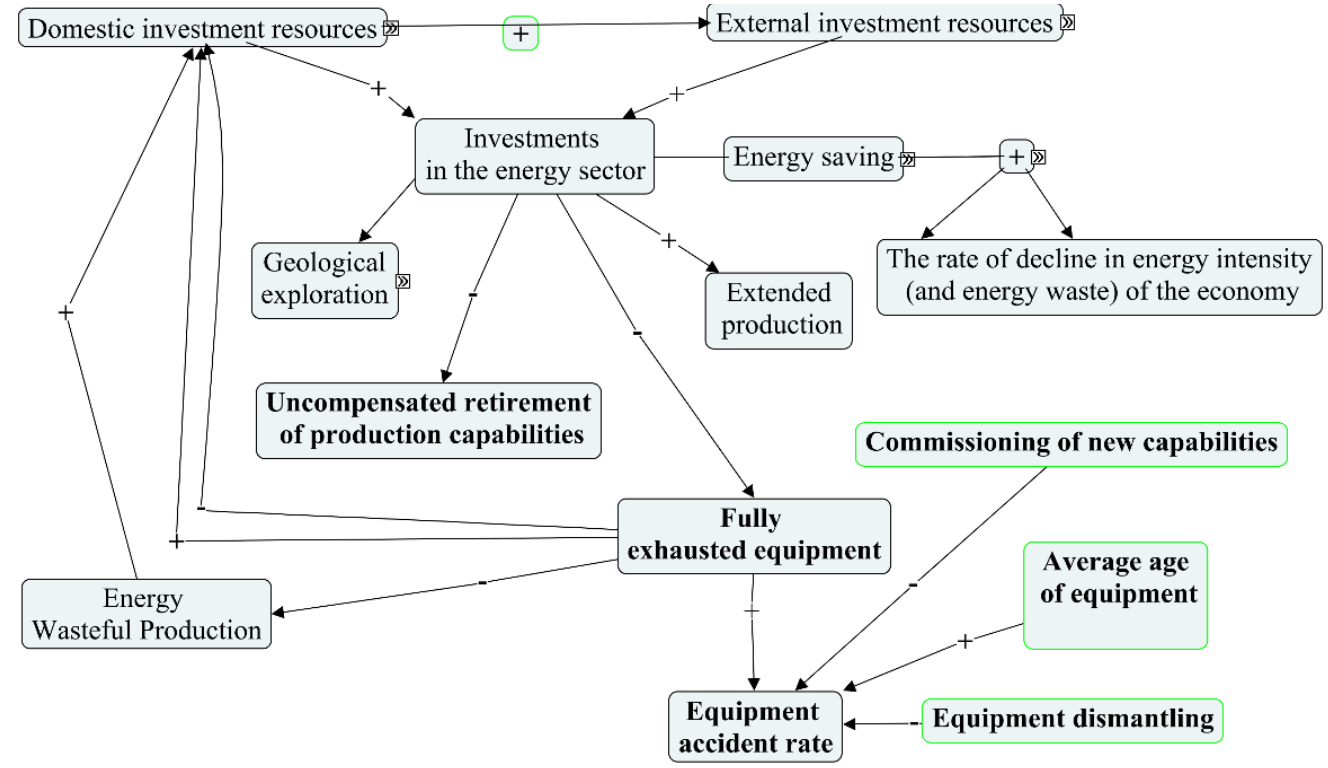

Fig. 3. Cognitive map Fragment of the threat to energy security "Lack of Investment" 
Figure 4 illustrates the transition to dynamic cognitive maps. For that purpose, let us take basic concepts such as investments in energy, equipment generating a resource, and accident rates.

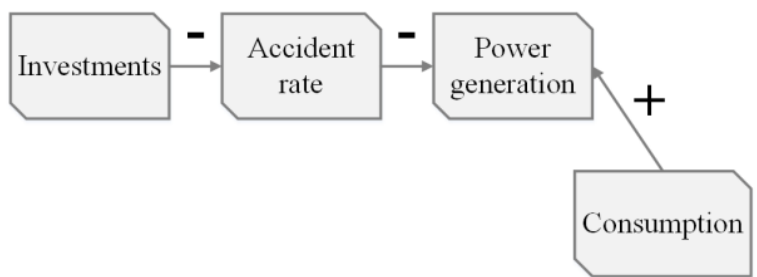

Fig. 4. A fragment of a cognitive map of the mutual influence of investments at electricity production

This illustrative example represents a generalized structure of TRV model as a set of following main characteristics (Table 1).

To go to dynamic fuzzy cognitive maps, we need to enter parameters for concepts. So, for example, the concept of "Power generation" has a set of some parameters. They are power parameter, which is measured in MWh, parameters that characterize the energy sector itself (name of the power plant, type of power plant, type of equipment). Besides each type of equipment contains its own attributes, for example, such as the name of the unit, unit capacity, technology, age.

The main difficulty in working with the TRV model is its orientation to the parameters of the object, and not to their aggregation and representation of the object behavior. Figure 5 shows an example of detailing the concepts of the mutual influence of investments on electricity production and shows the relationship between the parameters of the concepts of "Accident rate" and "Block" of energy facilities.
TABLE I. DESCRIPTION OF CONCEPTS

\begin{tabular}{|l|l|l|}
\hline \multicolumn{1}{|c|}{ Concepts } & \multicolumn{1}{|c|}{$\begin{array}{c}\text { Bunch of } \\
\text { parameters }\end{array}$} & \multicolumn{1}{|c|}{ Characterization } \\
\hline Investments & $\begin{array}{l}\text { Commissioning } \\
\text { of new } \\
\text { capacities (cu) }\end{array}$ & Financial limitation \\
\cline { 2 - 3 } & $\begin{array}{l}\text { Dismantling of } \\
\text { old capacities } \\
\text { (cu) of }\end{array}$ & $\begin{array}{l}\text { Failure probability } \\
\text { limit (\%) }\end{array}$ \\
\cline { 2 - 3 } Accident rate & $\begin{array}{l}\text { Probability } \\
\text { failure (\%) }\end{array}$ & $\begin{array}{l}\text { Disabled capacity } \\
\text { limit (MWth) }\end{array}$ \\
\hline Power \\
generation & $\begin{array}{l}\text { Power of block } \\
\text { (MWth) }\end{array}$ & $\begin{array}{l}\text { Total capacity } \\
\text { constraint by region } \\
\text { (MWth) }\end{array}$ \\
\hline Consumption & MWh & $\begin{array}{l}\text { Short supply limit } \\
\text { (MWh) }\end{array}$ \\
\hline
\end{tabular}

Limitations and parameter values are known from the model. Thus, if we set all these parameters in the dynamic fuzzy cognitive maps means and add restrictions to them, then the expert is given the opportunity to make changes to the mathematical model.

The architecture of the IT environment was previously proposed to ensure the application of dynamic cognitive maps in this application area [12]. Further research is related to the development of interfaces for the study of energy development directions taking into account energy security in accordance with the TRV model.

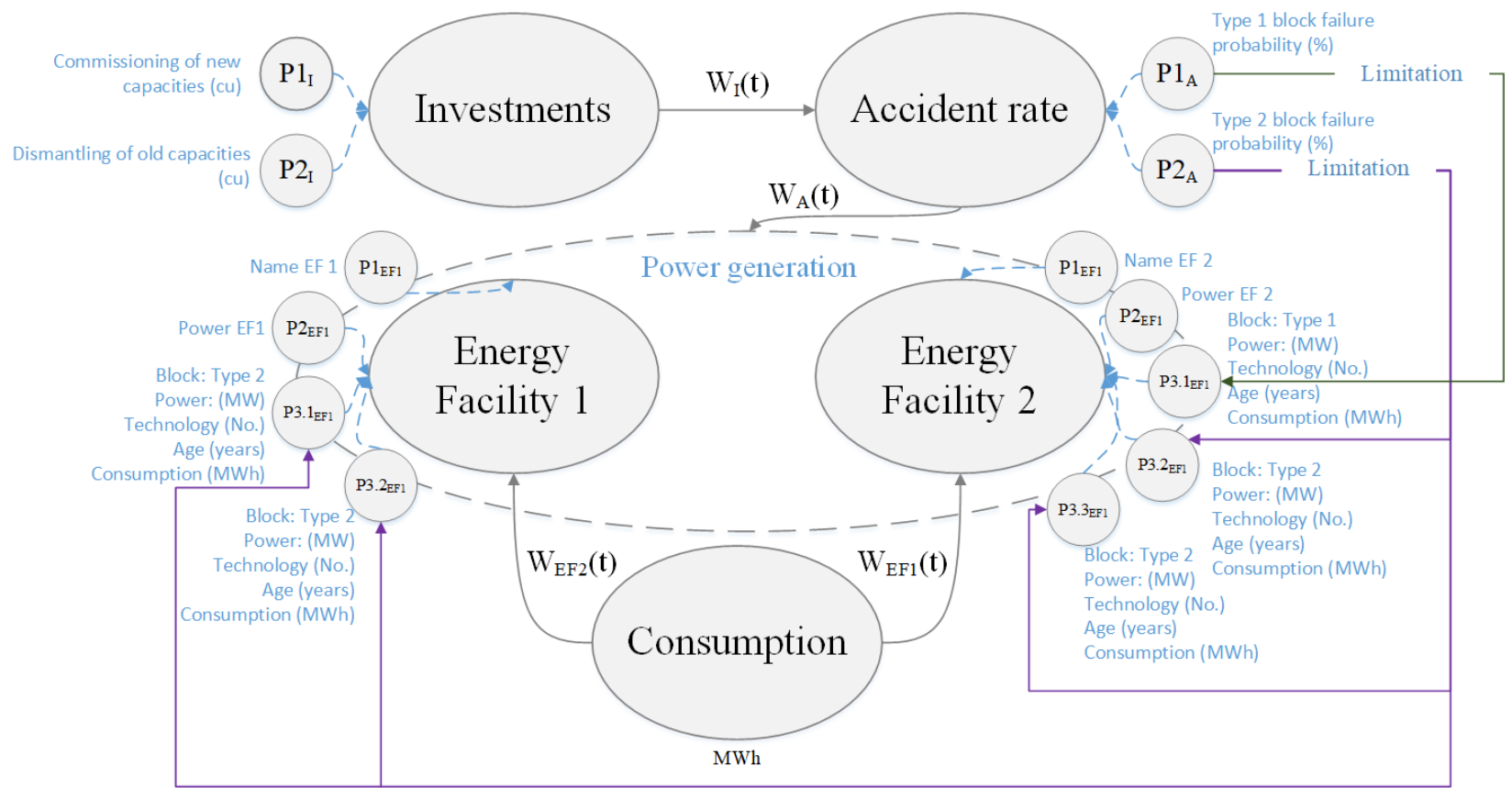

Fig. 5. Illustrative example of a dynamic fuzzy cognitive map application to present the mutual influence of investments at electricity production 


\section{CONCLUSION}

The article considers the dynamic cognitive maps as a means of visual analytics and cognitive graphics. The use of dynamic cognitive maps in studies of the energy sector development from the standpoint of energy security will allow working with the traditional mathematical model not directly but through visualization tools. The presented situations in the form of a graph and binding to real energy facilities allow us to work directly with the mathematical model of the energy sector. Moreover, there are two possible options. Firstly, the visualization of a computational experiment using dynamic cognitive maps, and the display of results in dynamics. Secondly, experts have the opportunity to adjust the model and set parameters, including emergency situations, directly through a dynamic cognitive maps interface.

According to the authors, this approach should significantly reduce the complexity of the computational experiment, as well as, through visualization, increase the level of perception of the results.

\section{ACKNOWLEDGMENT}

This work was performed within the framework of project according to state assignment MESI SB RAS №AAAA-A17-117030310444-2. The study of separated aspects was supported by RFBR grants № 19-07-00351, № 19-57-04003, № 18-07-00714, № 1837-00271, № 18-57-81001, № 17-07-01341.

\section{REFERENCES}

[1] Cui W. "Visual Analytics: A Comprehensive Overview" (2019). Vol. 7, IEEE Access, 2019. pp. 81555- 81573. doi:10.1109/access.2019.2923736

[2] Massel L.V. "Ontological engineering and knowledge management to support strategic decision making on the development of smart energy" In: Proc. of XX All-Russian Conference Enterprise Engineering and Knowledge
Management (EE\&KM), PRUE, Moscow, Russia, 2017, pp. 59-65.

[3] Zenkin A.A. "Cognitive computer graphics". Pospelov D.A., ed. Nauka, 1991, 192p.

[4] Pospelov D.A. "Cognitive graphics as a window into a new world”. Software \& Systems, 1992, pp. 4-6.

[5] Simoff S.J., Böhlen M. H., and. Mazeika A, Eds. "Visual Data Mining: Theory, Techniques and Tools for Visual Analytics". Heidelberg, Germany: Springer-Verlag, 2008.

[6] Thomas JJ, Cook CA. "Illuminating the Path: The Research and Development Agenda for Visual Analytics". Los Alamitos: IEEE Computer Society, 2005, pp. 1-180.

[7] Keirn D, Andrienko G, Fekete J, Gorg C, Kohlhammer J, Melancon G. "Visual analytics: Definition, process, and challenges". In: Kerren A, ed. Proc. of the Information Visualization. LNCS 4950, Berlin: SpringerVerlag, 2008, pp. 154-175.

[8] Massel L.V., Massel A.G. "Semantic technologies based on the integration of the ontology, cognitive and event modeling". In: Proc. of Open Semantic Technologies for Intelligent Systems (OSTIS-2013), Minsk, Belarus 2013, pp. 247-250.

[9] Massel L.V., Massel A.G. Ivanov R.A. "Cognitive graphics and semantic modeling for geospatial solutions in energy sector". In: Proc. of the 21st international conference Territories' Sustainable Development: Cartography and GI Support, Krasnodar-Fiji, Russia, 2015, pp. 496-502.

[10] Ferreira Danger E.V., Terelyansky P.V. "Visual representation of cognitive maps in three-dimensional space". Research and Innovation, No. 3, 2013, pp. 106-110.

[11] Gaskova D., Massel A. "Semantic modeling of cyber threats in the energy sector using Dynamic Cognitive Maps and Bayesian Belief Network". In: Proc. of the 7th Scientific Conference on Information Technologies for Intelligent Decision Making Support (ITIDS 2019). Publisher: Atlantis Press, 2019. pp. - 326-329. doi: 10.2991/itids-19.2019.58.

[12] Massel A.G. "Dynamic Cognitive Maps for the Substantiation of Strategic Decisions on Management of Energy Sector Development". Industry 4.0, Issue 4. 2018, Publisher: Scientific Technical Union of Mechanical Engineering “Industry 4.0", pp. 190-193.

[13] Arshinsky V.L., Massel A.G., Fartyshev D.A. "Multiagent software complex for research of the energy security problem". In: Proc. of the XIV Baikal All-Russian Conference "Information and Mathematical Technologies in Science and Management". Vol. 3, 2009, Irkutsk, MESI SB RAS, pp. 283-289. 